\title{
Clinical Management of COVID-19: Conceptual Framework
}

Jordi Rello ${ }^{1-3}$, Ricardo Serrano4

1 Centro de Investigación Biomédica en Red de Enfermedades Respiratorias (CIBERES), Instituto Salud Carlos II, Madrid, Spain

2 Clinical Research in Pneumonia \& Sepsis (CRIPS), Vall d'Hebron Institute of Research, Barcelona, Spain.

3 Clinical Research, CHU Nimes, University Montpellier - Nîmes, Nîmes, France.

${ }^{4}$ Critical Care Department, Hospital de Hellin, Albacete, Spain.

I $\mathrm{n}$ the treatment of COVID-19, attention is focused on expensive, high technology equipment such as sophisticated ventilators or extracorporeal membrane oxygena- tion (ECMO), which requires highly trained health care workers and aggressive pharmacological measures with weak evidence of efficacy (1). However, low-cost, primary health care has a higher life-saving potential.

The most common mistake is to consider and manage the disease like an invasive pneumococcal infection which is complicated by an acute respiratory distress syndrome. All physicians are familiar with patients developing pneumonia that could cause septic shock with sudden onset leading to early admission to a hospital and development of ARDS (Acute Respiratory Distress Syndrome). However, the paradigm regarding COVID-19 is different. COVID-19 starts like a flu-like illness that is being complicated by "atypical pneumonia" with persistent fever and late onset (average 7-10 days) of hypoxemic respiratory failure (2).

To avoid neglect, we recommend a strategy based on effective interventions that should have a potential population impact. Most deaths do not occur in the intensive care unit (ICU). For each death in the ICU, there are three deaths in the emergency department (ED) and six deaths in hospital wards, because they are flooded with patients with rapidly progressing acute hypoxemia.

Good quality and feasible clinical care should be based on clear and simple protocols, user-friendly, implemented in hospital wards. We do not need severity-of-illness compound scores, such as CURB65 or SOFA, because all patients arriving at the ED will have the same score. Monitoring these patients with venous oxygen saturation and respiratory rate is the core of management. Aggressive control of the hyperpyrexia to prevent the endothelial injury associated with the cytokine storm is mandatory. Most of these patients at the ED have hypercoagulability and present hypovolemia after high fever and inflammation. No furosemide should be prescribed and they need to be managed as hypovolemic.

\section{Corresponding Author: Jordi Rello \\ E-mail: \\ jrello@crips.es}

Received: April 5, 2020 Accepted: April 5, 2020

Published: April 8, 2020

Suggested citation: Rello J, Serrano R. Clinical Management of COVID-19:

Conceptual Framework. Infect Dis Clin Microbiol 2020; 1: 46-47.

DOI: $10.36519 / \mathrm{idcm} .2020 .0003$ 
Furthermore, the central role of the oxygen needs to be detailed. These patients need to receive adequate supplies of oxygen to maintain $\mathrm{SaO}_{2}$ above $92 \%$. Non-invasive ventilation is contraindicated, because it delays intubation with the toll of causing acute lung injury. Artesanal systems, like adding a PEEP valve to a modified nebulization mask system ("Boussignac device") are useless because only obese patients with sleep apnea take benefit, with risk of sudden respiratory arrest in case of accidental withdrawal. Most patients should be maintained oxygenated with high concentration oxygen delivered by reservoirs' masks or high-flow nasal cannula (HFNT).

Given that the suggestion for prone positioning of the patient is almost unanimous, and that this task is relatively simpler in the non-intubated patient without receiving mechanical ventilation, the decision about prone positioning (with HFNT or oxygen reservoir) could be life-saving.

Patients often maintain severe hypoxemia, with lung opacities, and severe $\mathrm{PaO}_{2} / \mathrm{FiO}_{2}$ below 100 $\mathrm{mmHg}$, with a relatively good clinical tolerance. If the respiratory rate is maintained under $30 \mathrm{bpm}$, and the patient does not develop signs of muscle fatigue, intubation should not be indicated, and most of these patients will present improvement within 24-48 hours. Wait and see. However, if the patient is developing progressive hyperventilation (respiratory alkalosis with superficial respiration), she/he should undergo immediate intubation.
When the patient with COVID-19 is intubated, he is characterized by hypoxic vasoconstriction associated with severe hypoxemia. In this scenario, inhaled nitric oxide (or prostacyclin) should be prescribed as rescue therapy. Most of these patients do not need to be managed as an ARDS. Recruitment manoeuvres are not beneficial at all, and they are contraindicated. Prone positioning alternating with supine cycles will be of little benefit, only contributing to increase contamination risk of healthcare workers and increase workload and fatigue. It should be measured the lung compliance as core management. Two-thirds of COVID-19 patients will have high values $\left(>40 \mathrm{ml} / \mathrm{cm} \mathrm{H}_{2} 0\right.$ ) being likely that it represents hypoxemic pneumopathy without acute lung injury. The target should be to maintain a $\mathrm{PaO}_{2}$ level above $60 \mathrm{~mm} \mathrm{Hg}(8 \mathrm{kPa})$, with mild sedation (and relaxation if required) whereas PEEP levels should be maintained not higher than 8-10 $\mathrm{cm} \mathrm{H}_{2} \mathrm{O}$. Higher PEEP levels will compromise cardiovascular stability, requiring to add vasopressors, without respiratory benefit and with the risk of acute kidney injury. Respiratory rate can be lower than $20 \mathrm{bpm}$ and tidal volumes above $6 \mathrm{ml} / \mathrm{Kg}$ should be allowed.

In summary, severe COVID-19 is not an invasive bacterial pneumonia and SARS is not ARDS. Reducing the burden of limited ICU capacity by providing good quality essential health care depends on correct oxygenation strategies in the ED and hospitalization wards. Right oxygenation and respiratory management is the mainstream of life-saving interventions in the treatment of COVID-19 patients.
Peer-review: Externally peer-reviewed.

Conflict of Interest: The authors have no conflict of interest to declare.
Financial Disclosure: The authors declared that this study has received no financial support.

\section{REFERENCES}

1 Rello J, Tejada S, Userovici C, Arvaniti K, Pugin J, Waterer G. Coronavirus Disease 2019 (COVID-19): A critical care perspective beyond China. Anaesth Crit Care Pain Med 2020; https:// doi.org/10.1016/j.accpm.2020.03.001. [Epub ahead of print]..
2 Barrasa H, Rello J, Tejada et al. SARS-Cov-2 in Spanish Intensive Care: Early experience with 7-day mortality In Vitoria. Anaesth Crit Care Pain Med 2020. [In Press]. 